\title{
Application of Deep Learning Algorithms in Determination of Trace Rare Earth Elements of Cerium Group in Rocks and Minerals
}

\author{
Sumin $\mathrm{Ma}^{1,2}$ and Wenhui Huang $\mathbb{D}^{1,3}$ \\ ${ }^{1}$ School of Energy Resource, China University of Geosciences (Beijing), 100083 Beijing, China \\ ${ }^{2}$ Key Laboratory of Marine Reservoir Evolution and Hydrocarbon Enrichment Mechanism, Ministry of Education, \\ 100083 Beijing, China \\ ${ }^{3}$ Beijing Key Laboratory of Unconventional Natural Gas Geological Evaluation and Development Engineering, 100083 Beijing, China
} Correspondence should be addressed to Wenhui Huang; huangwh@cugb.edu.cn

Received 24 March 2021; Revised 17 April 2021; Accepted 20 May 2021; Published 28 June 2021

Academic Editor: Wenqing Wu

Copyright (c) 2021 Sumin Ma and Wenhui Huang. This is an open access article distributed under the Creative Commons Attribution License, which permits unrestricted use, distribution, and reproduction in any medium, provided the original work is properly cited.

\begin{abstract}
Since the breakthrough of deep learning in object classification in 2012, extraordinary achievements have been made in the field of target detection, but the high time and space complexity of the target detection network based on deep learning has hindered the technology from application in actual product. To solve this problem, first of all, this paper uses the MobileNet classification network to optimize the Faster R-CNN target detection network. The experimental results on the rare earth element detection data set show that the MobileNet classification network is not suitable for optimizing the Faster R-CNN network. After that, this paper proposes a classification network that combines VGG16 and MobileNet, and uses the fusion network to optimize the Faster R-CNN target detection network. The experimental results on the rare earth element detection data set show that the Faster R-CNN target detection network optimized by the fusion classification network has the advantages of using VGG16 and MobileNet's Faster R-CNN target detection network to detect rare earth elements. The innovation of this article is that the results on 5 time series data sets show that CDA-WR has better predictive performance than other ELM variant models. The effect of determining trace cerium elements in rocks and minerals is increased by more than $50 \%$, based on deep learning. The algorithm studies the methods of target detection and recognition and integrates it into the intelligent robot used in this subject, giving the robot the ability to accurately detect the target object in real time.
\end{abstract}

\section{Introduction}

Adding trace amounts of cerium to steel for offshore platforms is expected to replace the more expensive titanium and vanadium elements and reduce costs. This is also in line with my country's resource characteristics (the properties and functions of cerium in the smelting, processing, and alloying of steel are relatively similar), cerium reserves are abundant, and the price is relatively low. It can be used to improve the quality of steel for offshore platform structures. Optimize the smelting, plastic processing, and heat treatment processes of rare earth steel with trace cerium, and it is expected that trace cerium will be used to improve the perfor- mance of rare earth steel for offshore platforms. Compared with other alloying elements in steel, rare earth elements have a larger atomic radius, ranging from $0.161 \mathrm{~nm}$ to $0.204 \mathrm{~nm}$, which is about $50 \%$ more than iron atoms $(0.117 \mathrm{~nm})$; this makes rare earth elements easy to lose their outer electrons. As cations are formed, their electronegativity is very low, which is only slightly higher than that of alkali metals and alkaline earth metals of the same period; this also makes rare earth elements exhibit extremely strong reactivity in molten steel, and they are not only easy to interact with molten steel. Oxygen, sulfur, and other nonmetallic elements combine to form high melting point rare earth compounds. At the same time, rare earth elements can interact with many low melting 
point metals in steel, thereby reducing their harm to the mechanical properties of materials.

Shugui and Wang used the least squares regression algorithm to process the data by spectrophotometry with the same hair color and tribromide system to simultaneously identify rare earth cerium in geological samples and achieved satisfactory results. Discuss in the sample, determine the composition of the calibration sample group through the proposed method, determine the best factor and the GSD and GSR series of the national standard geological sample series, and the overall results are consistent with the recommended values. However, they took very few samples and cannot generalize [1]. Yan and Zhijie proposed an SSD deep learning algorithm to improve the performance of multiobject protocols. Three different sample projects were trained. Firstly, the SSD neural network algorithm detects the type of object and creates a delimiter around the location of the object in the image. Secondly, find the smallest rectangle around the object according to the contour feature of the object and calculate the position coordinates of the object in the image. Finally, complete the handle configuration design information to prevent the handle from colliding with other objects and causing the handle to fail, but the algorithm is not complete, and the actual effect is not ideal [2]. Jun et al. believe that as the demand for oil and natural gas resources continues to grow, it is necessary to strengthen technological innovation in oil and natural gas exploration. Therefore, more effective seismic data processing technology is needed. The increase in computer performance has led to an explosive growth in the field of deep learning. The exploration field has also been extensively developed. Deep learning is a machine learning method. Its development has experienced two waves, namely, BP algorithm and deep learning. The applications listed below were also created in these two waves. Deep learning is a type of supervised learning, which allows to learn more advanced abstract representations of data and automatically extract features from the data. But deep learning needs to be more intelligent, and they did not solve the problem of deep learning intelligence [3].

To study the effect of trace rare earth element cerium (ppm level) on the structure and properties of steel, the steel used for offshore platforms has higher requirements for impact toughness in addition to the conventional mechanical performance test. Denatured inclusions (sulfides) change the strip-shaped manganese sulfide into spherical or ellipsoidal rare earth sulfides, which is beneficial to improve the plastic toughness of steel. Study the influence and effect of the trace rare earth element cerium on the CCT curve, Ac1 and Ac3 points of the experimental steel, and use the end quenching experiment to determine the hardenability of the steel [4]. In microstructure organization analysis mechanical properties, analyze the influence of trace rare earth element cerium. The rare earth element cerium improves the seawater corrosion resistance of steel. Rare earth sulfide has a more positive electrode potential than manganese sulfide. Due to the reduced potential difference and the reduction of the galvanic effect, the presence of rare earth sulfide in the grain boundary is beneficial to improve the steel corrosion resistance of the substrate.

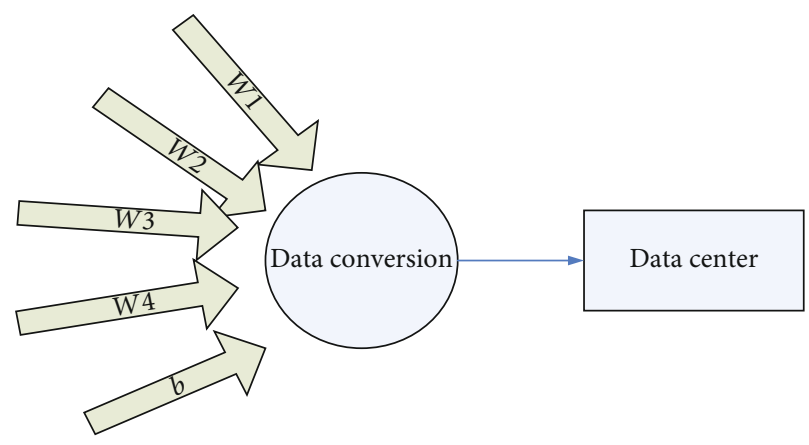

FIgURE 1: Neuron model.

The corrosion resistance of the high-strength steel used in seawater solution was analyzed [5].

\section{Deep Learning Algorithms Are Measuring Rock and Mineral Methods}

2.1. Traditional Target Detection Algorithm. Target detection is an important problem in computer vision, and many researchers have developed a large number of algorithms. The general flow of the traditional target detection algorithm is shown in Figure 1. The main flow is to first perform redundant window interception through a sliding window to ensure that the target is contained by at least one window. Due to the various sizes and aspect ratios of the target, the interception window also uses preselected windows of different sizes and aspect ratios for interception [6, 7]. With the intercepted image, it is necessary to perform preprocessing operations, including unifying the size, removing the mean value, eliminating irrelevant features, and reducing noise. Then, a series of images after processing and processing are used for feature extraction using artificially designed feature operators. Feature selection is the key to the problem of target detection, and the quality of features can often determine the performance of the final target detection algorithm to a large extent. Different tasks often require different requirements for features, so there is no universal and universal feature. Commonly used traditional feature extraction methods include shape, color, texture, edge, corner, and other visual features, as well as better performance of artificially designed feature operators, such as HOG features, SIFT features, LBP features, and Haar wavelet feature operators. After feature extraction, classifiers commonly used in machine learning, such as SVM and AdaBoost, are used for classification and regression, and finally, the detection results are generated.

The basic computing unit of artificial neural network is called perceptron, also known as neuron, which was first developed by Rosenblatt in 1957 [8, 9]. It is a simplified human neuron cell model. It is essentially a function. It accepts multiple real number input variables. Each variable has a weight value on the connection. The input variable is added to a bias term after weighted summation. As the input of the neuron, it passes through a linear activation function. The linear activation function finally produces an activation value [10]. The input value simulates the stimulation of nerve 
cells, and the output value simulates the response of nerve cells. The neuron model is shown in Figure 1:

2.2. Deep Learning Algorithm. When using deep learning in real life, the first thing to do is to optimize the network. The training process includes layer-by-layer training for unsupervised learning and network optimization for supervised learning. Through sampling and training the network, they found the inherent rules and used it to improve the user's network performance $[11,12]$. To improve network performance, it must be achieved through specific learning steps. The three common learning steps that are commonly used are presented as follows:

2.2.1. Supervised Learning. A common method of machine learning is supervised learning, which means that external monitoring methods are used in network learning to reward good learning, punish misleading learning, and always correct errors in the learning process. At the same time, the training pattern used in this learning method must be recognized as a pattern $[13,14]$. In order to make training more appropriate, the training model should cover as many situations as possible. Supervised learning includes inputting samples into the network, obtaining network labels through step-by-step learning, then comparing the obtained network labels with the control group, and finally changing the network parameters according to the supervised learning criteria so that the network labels are similar to the sample labels $[15,16]$.

2.2.2. Unsupervised Learning. Unsupervised learning is different from supervised learning. Unsupervised learning has no external monitoring function. The learning system is very smart. It will add to its own database and make statistics based on the data entered by the user, change the network label according to the internal changes in the database, and finally complete the upgrade of the network. This learning method is often used to learn labels and collect data $[17,18]$.

2.2.3. Reinforcement Learning. Learning aid is an ongoing Markov decision-making process. In the process of online training, there are no formal results like supervised learning. After each online training course, only one evaluation function is provided. The network usage evaluation function evaluates learning outcomes. In addition to evaluation, network parameters must be adjusted according to the evaluation results, and finally, the entire network evaluation function is optimized $[19,20]$.

In the actual application of neural networks, it is necessary to choose appropriate learning methods for different application fields. The most important thing is that the three proposed learning methods have strong learning ability [21]. However, generally speaking, supervised learning algorithms are mainly used to optimize and adjust network parameters, and unsupervised learning algorithms are mainly used to classify multiple samples. Reinforcement learning algorithms are mainly used for intelligent control, analysis, and prediction.

\section{Correlation Experiment of Rare Earth Elements of Cerium Group in Rocks and Minerals}

The location of the rare earth element lanthanum and cerium in steel is very important. The segregation at grain boundaries or the amount of solid solution in the grain will have a great impact on the quality of steel. Therefore, it is necessary to explore the location of trace amounts of lanthanum and cerium. In the neuron model shown, $X$ is the input variable of the neuron, representing a sample defined by four attribute values $[22,23] . W$ represents the corresponding weights of the four attribute values on the neuron, $b$ is the bias term of the neuron, and the activation function used is the hard limiter. The function expression is as follows:

$$
f(x)=\left\{\begin{array}{cc}
1 & x>0 \\
-1 & \text { otherwise }
\end{array}\right\} \text {. }
$$

The input value $Z$ of the neuron is the weighted sum of the bias item plus the attribute value and the weight:

$$
Z=\sum_{i=1}^{4} x_{i} y_{i+b}
$$

The neuron model can perform simple sorting and regression tasks, but cannot perform complex tasks [24, 25].

The activation function is an important part of the neuron, which is essentially a nonlinear function. It takes real numbers as input and produces real number outputs. Its main function is to improve the expressive ability of neurons and neural networks so that they can handle complex nonlinear problems. The first activation function used is a hard limiter, but it has many disadvantages. With the continuous development and improvement of artificial neural network theory, researchers have developed a variety of activation functions. Commonly used activation functions include sigmoid and hyperbolic tangent functions tanh and ReLU, Leaky ReLU, and maxout [26].

The sigmoid function accepts a real number and assigns it a number between $[0,1]$. Its expression is as follows:

$$
\varepsilon(x)=\frac{1}{1+e^{x}}+b .
$$

The double tangent function tanh accepts a real number and maps it to a number between $[0,-1]$, and its expression is as follows:

$$
\tanh (y)=\frac{e^{x}-e^{-x}}{e^{x}+e^{-x}}-\frac{1}{x}
$$

The powerful function of the deep learning system far exceeds the previous system. Based on the neural network and based on the huge network structure and huge data information, the number of hidden layers has increased $[27,28]$. Due to its excellent learning ability and random distribution, the application fields of deep learning are 


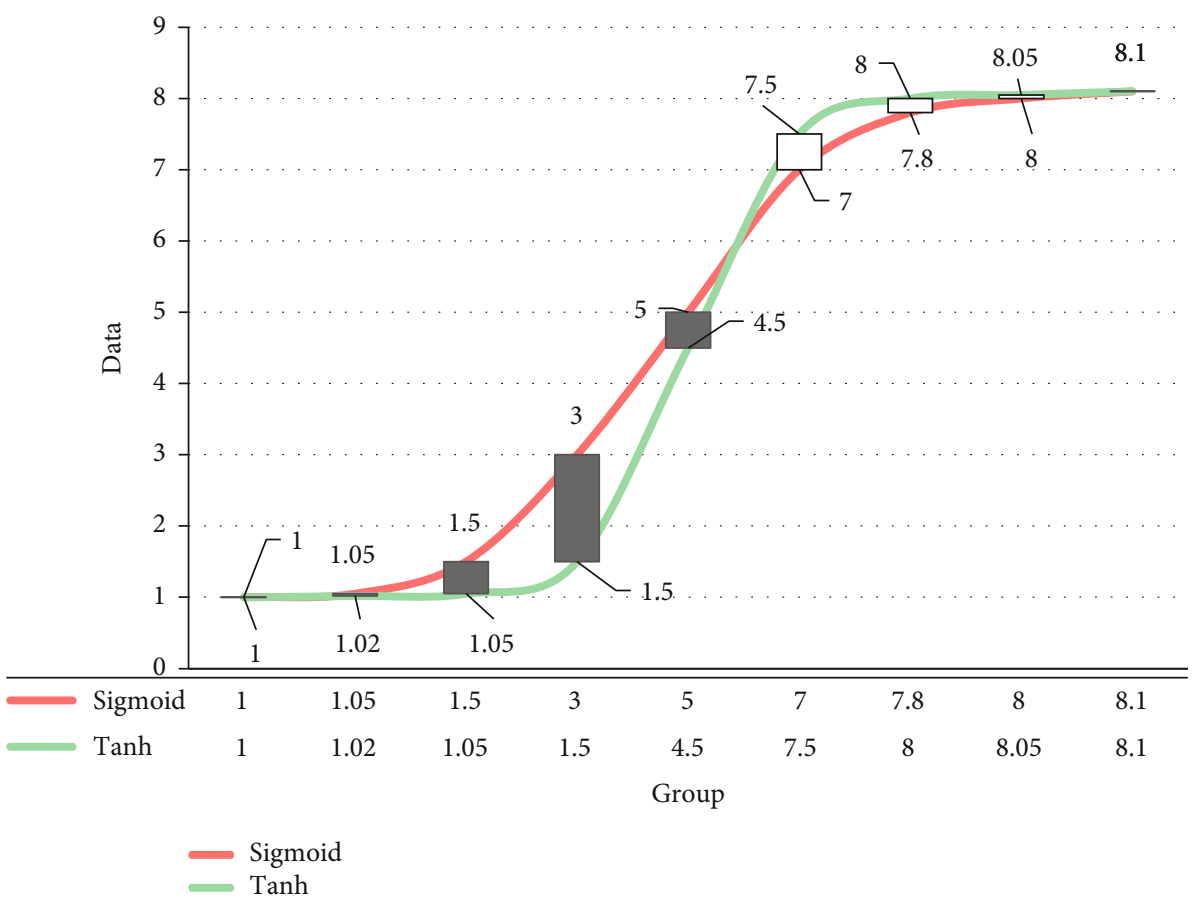

Figure 2: Sigmoid and tanh function images.

rapidly expanding. With the continuous expansion of deep learning research, deep learning algorithms for different fields are constantly being proposed. In order to make it easy to use, common deep learning algorithms and their respective application fields are given. Automated encoders and deep trust networks usually classify documents most effectively. For image classification, gravure network and convergent neural network are more suitable, but deep network is considered to be suitable for image recognition and classification, while convergent neural network is suitable for image recognition, and repetitive neural network is more suitable for speech recognition. For predictive analysis conducted in chronological order, the deep trust network can handle it well [29].

The curves corresponding to the two functions are shown in Figure 2. Their common feature is that the curve is symmetrical about a certain point, changes quickly near the symmetrical point, and smoothly changes away from the symmetrical point. These two activation functions have been widely used in early artificial neural networks, but there are problems in the back propagation process such as a large amount of calculation and vanishing gradient (vanishing gradient). These problems make the training efficiency of multilayer neural networks more efficient, low or no training at all. In response to these problems, new activation functions have been developed one after another [30] as shown in Figure 2.

ReLU (rectified linear unit) is an activation function developed by Vinod Nair et al. in 2010. Its function expression is as follows:

$$
f(x)=\max \left(\cos x * e^{x}, \int_{1}^{n} \sqrt[3]{x+b}\right)
$$

The output is 0 when $x<0$, and $x$ is output when $x>0$. Compared with sigmoid and tanh functions, ReLU can greatly accelerate the convergence speed of neural network training using the backpropagation algorithm, and because it does not involve complex exponential calculations, it reduces the amount of calculation.

$$
f(x)=\left\{\begin{array}{cc}
a e^{x}+b & x<0 \\
\sqrt{e^{x}} & \text { else }
\end{array}\right\} .
$$

Early artificial neural networks are mostly composed of fully connected layers; that is, any neuron in the current layer is connected to every neuron in the previous layer. The following figure shows a three-layer artificial neural network model constructed from fully connected layers:

$$
Q_{j}=\sum Q_{i j}+a_{j} Z_{k}=\sum Q_{j k} x_{j}+a_{k} r_{i}
$$

The batch stochastic gradient descent algorithm first divides the training set into $n$ batches; each batch contains a part of samples, and each update process uses only one batch of training data for parameter update. Mini-batch SGD is a generalization of the SGD algorithm. The original SGD is a special case of mini-batch SGD when $n=1$; that is, only one sample is used for each training. The literature also proves the convergence of the SGD algorithm. The expression of mini-batch SGD algorithm is as follows:

$$
\theta_{i}=\theta_{i+1}-\mu \bullet \varepsilon(\sin \alpha, \cos \beta)
$$

In addition to the parameter update algorithms introduced above, algorithms such as RMSprop, Adam, Adamax, and 
TABLE 1: Adding trace rare earth elements of cerium group to stainless steel.

\begin{tabular}{lcccc}
\hline Grade & After solution treatment $(b)$ & After solution treatment $(s)$ & $80 \%$ cold processed $(b)$ & $80 \%$ cold processed $(s)$ \\
\hline Modification 201 & 803 & 380 & 1890 & 1652 \\
$W(\mathrm{Cu})=1 \%$ & 726 & 360 & 1703 & 1503 \\
$W(\mathrm{Cu})=2 \%$ & 692 & 344 & 1620 & 1096 \\
$W(\mathrm{Cu})=3 \%$ & 663 & 335 & 1517 & 1121 \\
304 & 599 & 270 & 1415 & 1317 \\
\hline
\end{tabular}
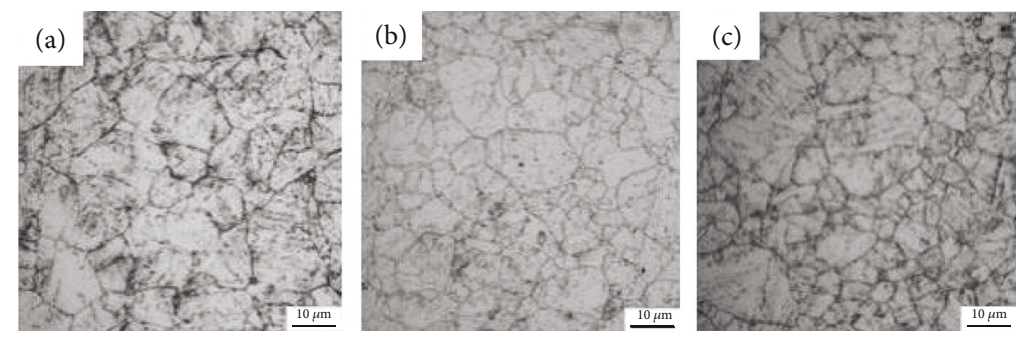

FIgURE 3: The optical morphology of original austenite of the three steels: (a) steel A, (b) steel B, and (c) steel C.

Nadam have been successively released. These algorithms ensure that deep learning models can be effectively trained. In summary, the matrix $f(x)$ required to complete the PCA dimensionality reduction goal is the matrix arranged in rows after unitizing the eigenvectors of the covariance matrix, where each row is an eigenvector of $x$. High-dimensional data uses the PCA algorithm to reduce the data to the $Q$ dimension, and the value of $Q$ needs to be determined by the percentage of the original information retained. Due to its special external electronic structure and very active chemical properties, rare earth elements will affect the thermodynamics, kinetics, crystallization process, and final electronic properties of the metallurgical steel grid during the solidification process. Compared with stainless steel without rare earth elements, the mechanical properties after adding rare earth elements have been significantly improved. The tensile strength is increased by more than $15 \%$, the yield point is increased by about $5 \%$, the impact strength is increased by more than $20 \%$, and the hardness is reduced by more than $170 \mathrm{HV}$. In addition, rare earths added to stainless steel can also play a role in improving oxidation resistance and hot workability.

\section{Experimental Analysis of Trace Rare Earth Elements in the Cerium Group}

In order to ensure the stability of the structure after adding trace elements of the rare earth element of the cerium group to the stainless steel, a method of reducing the content of nickel and carbon and increasing the content of manganese and nitrogen is generally used. On the one hand, it stabilizes the structure of austenite. On the other hand, stainless steel has good cold forming properties, which can ultimately reduce material costs and processing costs. In recent years, in order to improve and improve the corrosion resistance and cold workability of 200 series stainless steel, expand its application range, and achieve the purpose of replacing 304 in some application fields, the performance of 200 series stainless steel has been researched and improved mainly as shown in Table 1.

Adding copper elements and adding copper to stainless steel can greatly improve the corrosion resistance and can also increase the destructive power of the stainless steel laminate, improve the hardening effect that occurs during the cold forming process, and improve the cold forming efficiency. The research of adding copper to 201 modified stainless steel to make $204 \mathrm{Cu}$ stainless steel and replacing 304 stainless steel with this type of stainless steel is very successful. Due to the addition of copper to $204 \mathrm{Cu}$, the work hardening rate is significantly reduced, and the cold resistance is better than 304 .

Figures 3(a), 3(b), and 3(c) are the original austenite metallographic morphology diagrams of the three steel samples of A, B, and C, respectively. There are three statistics for each group of samples of A, B, and C. For the grain size data, the average size of the original austenite grains is shown in Tables 2 and 3. It can be seen from Tables 2 and 3 that the rare earth element cerium has no effect on the average size of the original austenite grains. The blank sample A, the sample $B$ with cerium, and the sample $C$ with cerium have no effect on the original austenite. The average size of the bulk crystal grains is basically the same. Although many documents report that the trace rare earth element cerium has the effect of refining crystal grains, the cerium added in this experiment formed a compound of cerium at about $10 \mathrm{ppm}$. Because the amount of cerium did not play a role in refining the crystal grains, it played a role in refining the crystal grains. The granular effect is still the fine-grained elements niobium, vanadium, and titanium. Marine engineering steel belongs to low-carbon microalloyed steel. Niobium, vanadium, and titanium are commonly used and effective elements for refining austenite grains in low- 
TABLE 2: The different cooling speeds of the phase transition temperature.

\begin{tabular}{lccccccr}
\hline Sample no. & & $\mathrm{A}$ & & & $\mathrm{B}$ \\
Organizational change & $\mathrm{A} \longrightarrow \mathrm{P}$ & $\mathrm{A} \longrightarrow \mathrm{B}$ & $\mathrm{A} \longrightarrow \mathrm{M}$ & $\mathrm{Mf}$ & $\mathrm{A} \longrightarrow \mathrm{B}$ & $\mathrm{A} \longrightarrow \mathrm{M}$ & $\mathrm{Mf}$ \\
40 & 669.5 & 431 & - & 295.6 & - & - & - \\
50 & 630.8 & 438.2 & - & 267 & - & - & - \\
60 & - & 478.1 & - & 259 & 450.3 & 436.3 & 275.2 \\
70 & - & 478.2 & 458.3 & 256.6 & 439.7 & 425.7 & 284.2 \\
80 & - & 486.7 & 466.9 & 266.4 & 413.1 & 401.9 & 249.4 \\
186 & - & 460.8 & 443.9 & 250 & 430 & 420.8 & 276.8 \\
\hline
\end{tabular}

TABLE 3: Test result statistics of the hardness of fracture surface.

\begin{tabular}{lcccc}
\hline Sample & 1.5 & 3 & 4.5 & 6 \\
\hline \multirow{2}{*}{ A } & 34 & 36.5 & 39 & 41 \\
& 37.5 & 38 & 38 & 41 \\
\hline \multirow{2}{*}{ B } & 36.5 & 37.5 & 41 & 39.5 \\
& 38.5 & 42 & 42 & 40.5 \\
\hline \multirow{2}{*}{ C } & 40 & 43 & 41 & 37 \\
& 38 & 42.5 & 41.5 & 41 \\
\hline
\end{tabular}

carbon microalloyed steels. Due to the high carbide stability of niobium and titanium, titanium is carbonized. The substance is slowly dissolved into the austenite when it is heated above $1000^{\circ} \mathrm{C}$, and the titanium carbide particles effectively prevent the austenite grains from coarsening. The pinning effect of vanadium on the growth of austenite is inferior to that of niobium and titanium.

According to the thermal expansion curve and metallographic analysis, when the cooling rate reaches $70^{\circ} \mathrm{C} / \mathrm{s}$, the metallographic structure is bainite and martensite. Since the transformation point of bainite to martensite cannot be distinguished in the expansion curve, the martensite is observed as variable temperature martensite from the expansion curve. The phase transition amount on the thermal expansion curve is approximately proportional to the temperature change.

As shown in Table 2, the cooling rates of samples $\mathrm{A}$ and $\mathrm{B}$ at $40^{\circ} \mathrm{C} / \mathrm{s}, 50^{\circ} \mathrm{C} / \mathrm{s}, 60^{\circ} \mathrm{C} / \mathrm{s}, 70^{\circ} \mathrm{C} / \mathrm{s}, 80^{\circ} \mathrm{C} / \mathrm{s}$, and $186^{\circ} \mathrm{C} / \mathrm{s}$ are combined with thermal expansion curve and gold CCT curve drawn by phase and hardness.

The line with triangles in the figure is sample $\mathrm{A}$, the line with squares is sample $\mathrm{B}$, and the dashed line is the transition line inferred from the experimental results. The pearlite transition zone combined with the slope of the thermal expansion curve of a- 40 and a- 50 in Figure 3 shows that at about $600^{\circ} \mathrm{C}$, sample A has an obvious phase transition process at $50^{\circ} \mathrm{C} / \mathrm{s}$; it can be seen from b- 40 at about $600^{\circ} \mathrm{C}$ that the turning line of sample $\mathrm{B}$ at $40^{\circ} \mathrm{C} / \mathrm{s}$ curve is not obvious, and very little pearlite transformation occurs. The "nose tip area" of the pearlite transformation of sample B with the addition of element lanthanum and cerium shifted to the right, which improved the stability of the supercooled austenite.

As shown in Table 3, when the rare earth element is added in a few hundred ppm, when it is combined with sul-

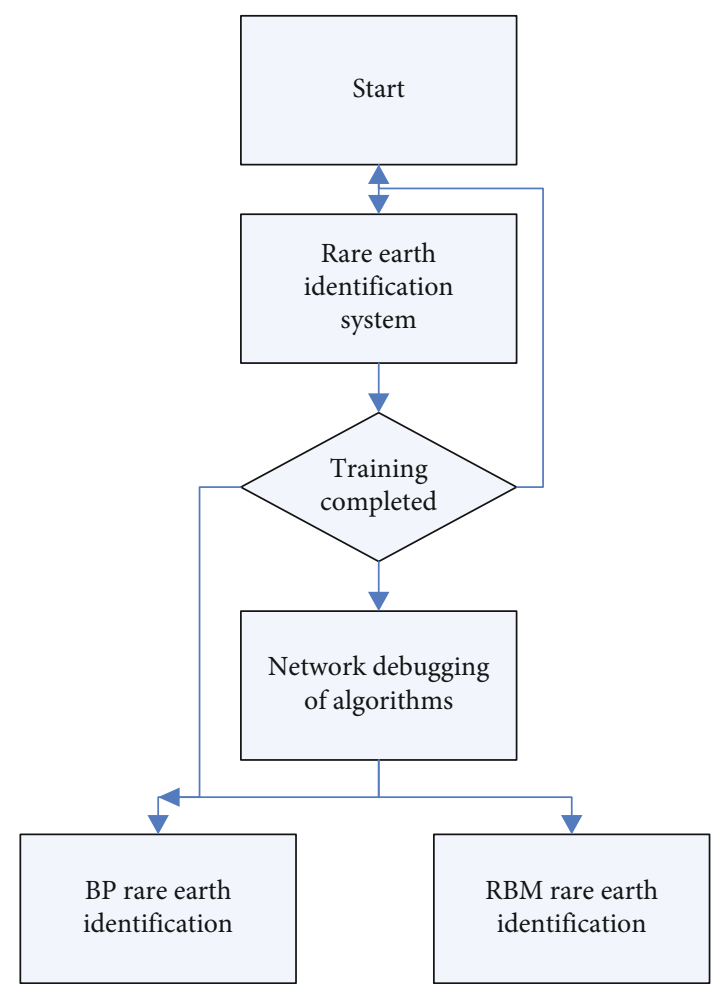

Figure 4: Test result statistics of the hardness of fracture surface.

fur, oxygen, etc., a part of it will dissolve in the matrix. When heated to austenitization, rare earth atoms will inevitably segregate to austenite grain boundaries. The segregation of rare earth atoms at the grain boundary inhibits the diffusion of carbon atoms to the grain boundary and delays the formation of cementite.

From Figure 4, we can see that when the training sample of the BP neural network increases, the learning ability and nonlinear mapping ability of the network decrease, which leads to the decline of the face recognition effect of the PCA-GA-BP algorithm. In order to overcome this shortcoming, this chapter replaces the BP neural network with the DBNs network with stronger learning ability to form the PCA-GA-DBNs network. The network first uses the PCA algorithm to process face images, reduces the amount of image data, and simplifies the network structure. During the corrosion process, the first part to be corroded is the 


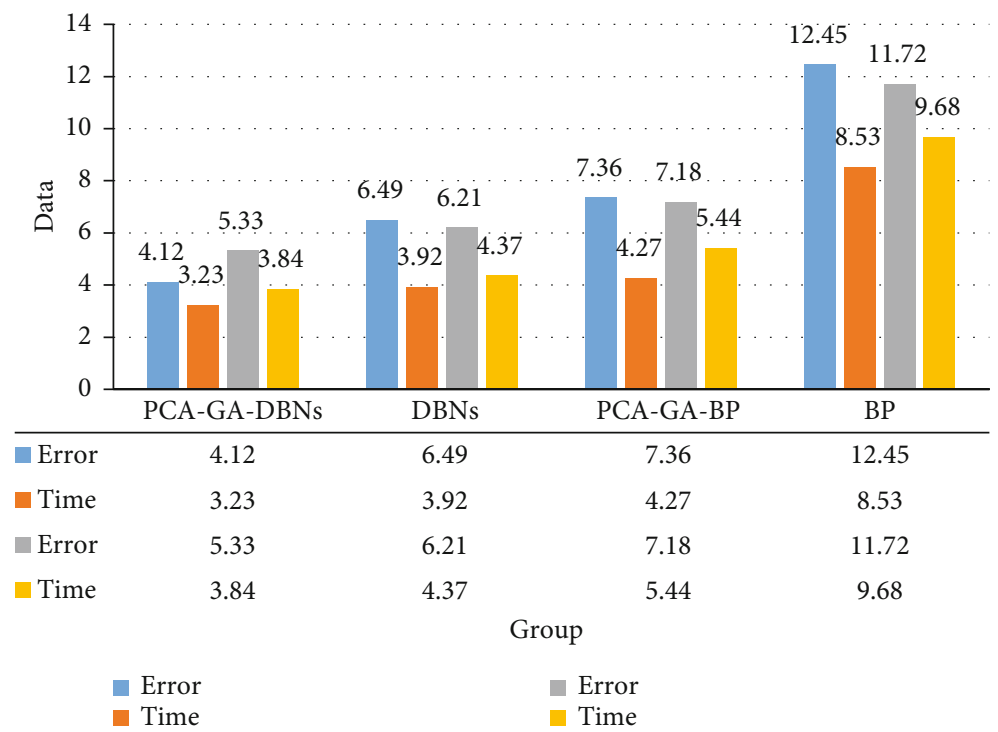

FIGURE 5: Recognition results of four comparison algorithms.

matrix around the elongated $\mathrm{MnS}$ inclusions, and as the corrosion progresses, a deep corrosion groove is formed on the periphery of the MnS. At the same time, the $\mathrm{MnS}$ inclusions themselves also appear a lot of dissolution. Increase the speed of corrosion pits to the surrounding development. In addition, $\mathrm{MnS}$ inclusions will be concentrated along the rolling direction during the rolling process, which seriously affects the mechanical properties of the experimental steel such as tensile strength and impact toughness.

It can be seen from Figure 5 that for all the test sets, the PCA-GA-DBNs network has the lowest average recognition error (4.61\%), followed by the DBNs network (6.69\%), PCA-GA-BP network (7.63\%), and BP neural network (12.59\%). And the recognition speed of the PCA-GA-DBNs network is significantly higher than other algorithms. The DBNs network is relatively close to the PCA-GA-BP network, and the BP neural network is the slowest. The PCAGA-DBNs network not only has the highest recognition accuracy but also has good recognition stability. For stainless steel, the number, composition, shape, and distribution of nonmetallic inclusions directly affect corrosion resistance and mechanical properties. After adding rare earth elements to stainless steel, the composition and morphology of inclusions change, and the overall performance of the stainless steel is improved. Generally, there are two methods to determine the critical point from the extension curve: the peak method (extreme value method) and the tangent method. When using the peak method, the extreme point with a clear slope in the extension curve is used as the critical point. The advantage of this method is that it is easy to determine the critical point, but the critical point determined by this method is not the actual critical point, and the set transition start temperature is higher than the actual critical point. The final transition temperature is lower than the actual temperature. The tangent method takes the boundary point between the extension line of the straight part of the expansion curve and the curve part as the critical point, which is closer to the actual starting temperature and ending temperature of the conversion. The sample inclusions are large composite rare earth inclusions composed of several smaller rare earth inclusions. The formation of such inclusions is due to the high adsorption of rare earth elements, which may lead to a high concentration of rare earth elements in the surrounding rare earth elements. Although this type of inclusion reduces the distribution of inclusions in the steel due to its larger size and uneven distribution and refines the steel to a certain extent, it has a significant impact on the performance of the tested steel. This experiment uses the tangent method.

From Figure 6, we can use BP network and RBM network to construct the PCA-GA-DBNs classifier and use it for face recognition. With the increase in the number of training samples, the recognition effect of each algorithm has been improved, but when the training sample is large, the face recognition accuracy of each algorithm has a small range of decline. Further analysis of the reasons can be obtained, GA algorithm in the case of a large number of training samples, the search range increases and the calculation amount increases sharply, its climbing ability is insufficient, and the defects that are prone to premature convergence are gradually revealed, thus slowing down the convergence speed of the network. This increases the probability of the network falling into the local optimum and affects the final face recognition result.

The main reason for the analysis is that the DBNs network is formed by superimposing multiple RBMs. The $\mathrm{RBM}$ is used to construct a classifier. During the training process, the data can be well connected from the hidden layer to the classification layer, and then, a good recognition effect can be obtained. For deep learning algorithms, different classifiers will get different recognition results. The main reason is that different classification algorithms have different training effects during network training, resulting in differences in 


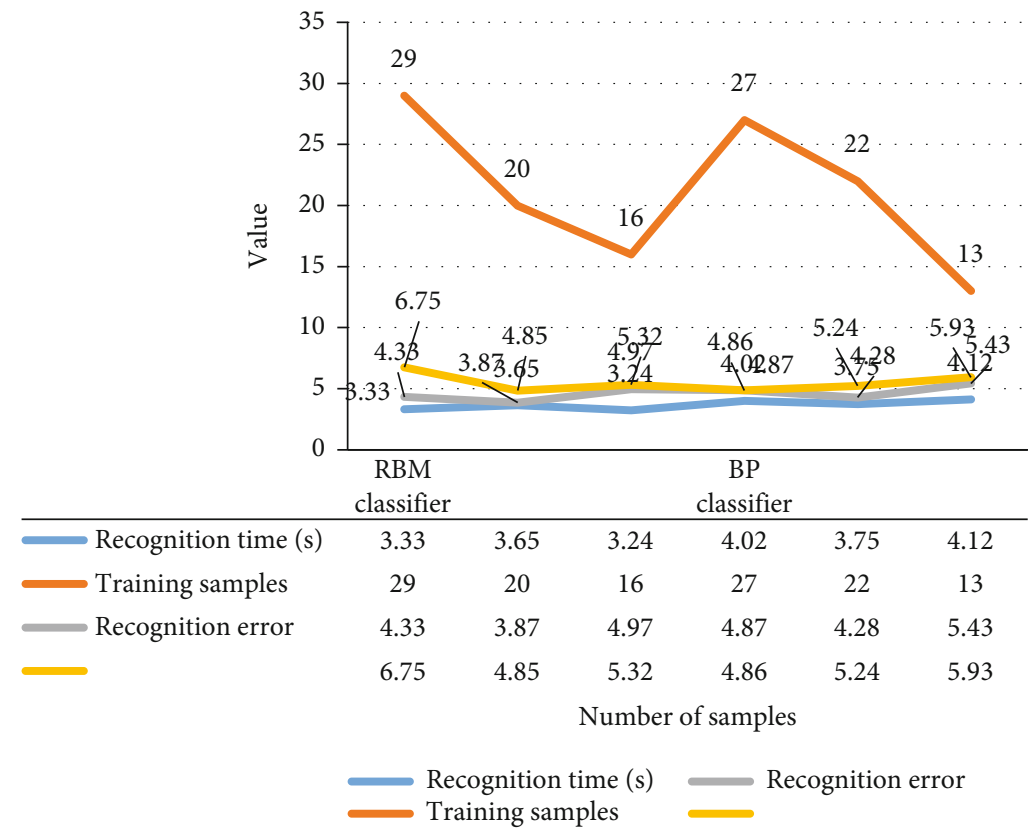

FIgURE 6: The average recognition error of the two classifiers.

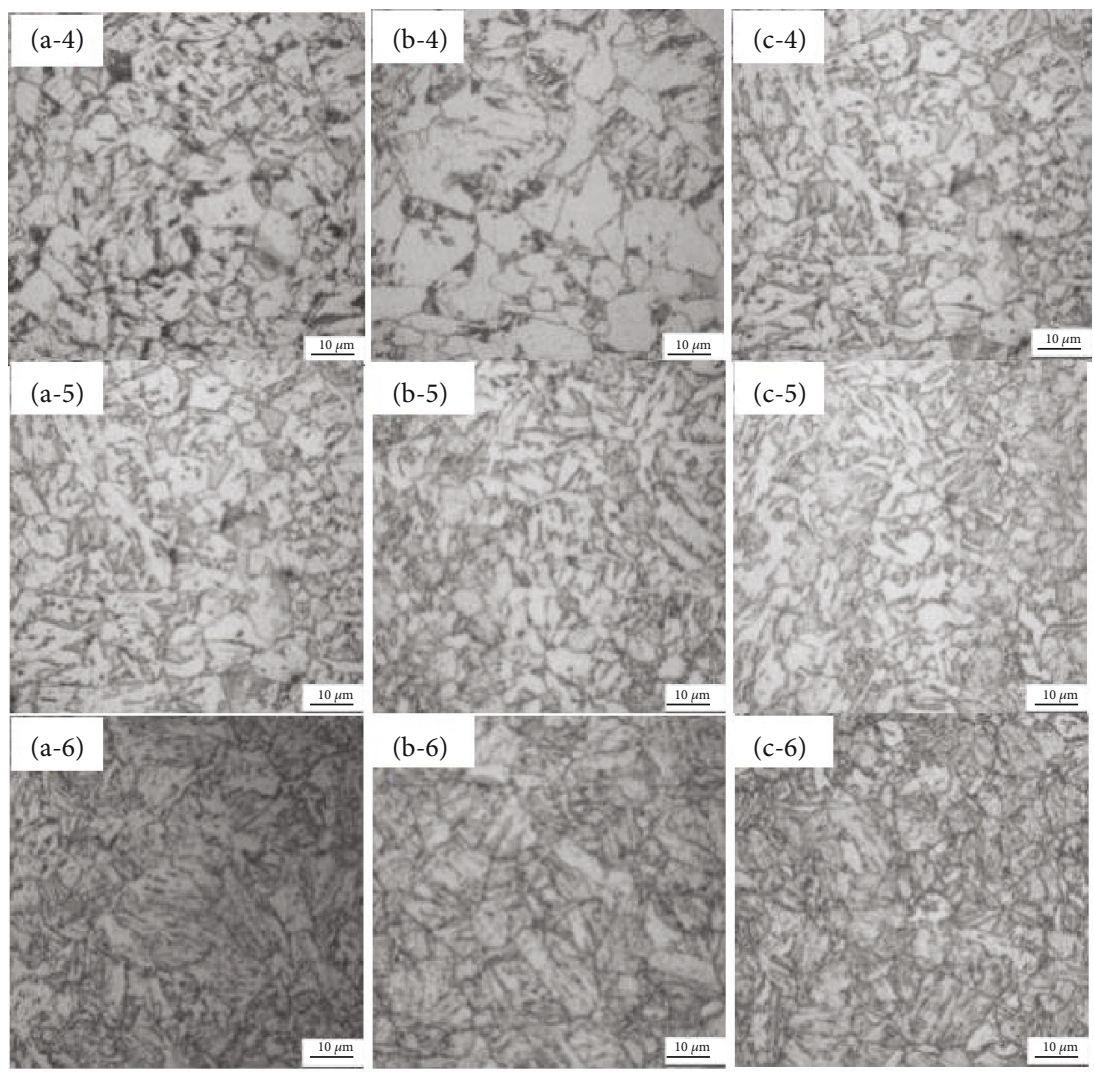

Figure 7: Microstructure normalizing (a-4), (b-4), and (c-4), subcritical quenching-tempering (a-5), (b-5), and (c-5), and quenchingtempering (a-6), (b-6), and (c-6).

classification capabilities between classification algorithms. But in general, after the classifier is fully trained, it can achieve better classification results.
Figures 7(a-4), 7(b-4), and 7(c-4) are the metallographic structures of the normalized state of the impact sample. The metallographic structure of the three is basically the 
same in morphology and size, and they are all iron element body pearlite tissue. Figures $7(a-5), 7(b-5)$, and $7(c-5)$ are the subtemperature quenched and tempered structure, the metallographic structure of the three is ferrite+ tempered troostite, and the ferrite grains tend to be multilateral because the subtemperature quenching temperature is in the twophase region of austenite and ferrite, partial austenitization, and the ferrite dissolved in the two-phase region has a cutting effect on the formed austenite grains and refines the austenite. The size of the tempered troostite is smaller than that of the tempered and tempered troostite. Figures 7(a-6), 7(b-6), and $7(c-6)$ in the quenched and tempered state are all tempered troostites. Under different heat treatment conditions, the metallographic structure of steel grades A, B, and C is basically the same. The addition of trace elements lanthanum and cerium does not change the structure of the steel, alters and changes the inclusions in the grain boundary, and improves the toughness.

Figure 7 shows the scanning morphology and energy spectrum of samples B and C. It has been determined by the nonaqueous electrolyte low-temperature electrolysis method that all lanthanum and cerium exist in the steel as inclusions. Observe the morphology of the inclusions modified by lanthanum and cerium. They are all round-shaped, and along with calcium, the elongated sulfides have been modified. (b-6) and (c-6) are the energy spectrum analysis of the inclusions. It can be inferred that the chemical composition of the inclusions is complex. Titanium can form $\mathrm{TiC}$, TiN, and (TixV1-x) N phases, and niobium can also generate carbon and nitrogen of niobium. It is possible that inclusions formed by calcium, lanthanum, cerium, and aluminum combine with niobium and titanium to form composite inclusions.

\section{Conclusions}

With the steady growth of deep learning, artificial intelligence has gradually invaded people's lives. As an important subject in the field of deep learning and computer vision, object recognition has gradually become the research focus of researchers. This article focuses on deep learning algorithms and points out that most deep learning algorithms use the same feature map to predict the two positions of prediction and category prediction. The prediction of the category requires different contradictions in the features of the feature map. In-depth target-based target research shows that the location prediction and category prediction in the target task put forward different requirements for the feature map function. For location prediction, the function map must contain a lot of detailed information and location information, while for category prediction, the function map needs abstract semantics: function and variability. However, existing algorithms use the same feature map to predict the two tasks of position prediction and category prediction. Target detection algorithms will play a huge role in the era of artificial intelligence. This paper studies the existing target detection algorithm and proposes several improvements to the SSD300 algorithm, which improves the performance of the algorithm to a certain extent, but there are still many problems that have not been solved. In the future, in-depth research will be conducted in the following areas. This article has improved the SSD network. The next step will try to apply the idea of separation of features to other target detection algorithm models based on deep learning and combine the characteristics of the algorithm to carry out targeted structural design for improvement.

\section{Data Availability}

No data were used to support this study.

\section{Conflicts of Interest}

The authors declare that they have no conflicts of interest.

\section{References}

[1] C. Shugui and Y. Wang, "Partial least squaresspectrophotometric determination of cerium group rare earth elements in geological samples," Rock and Mineral Testing, vol. 13, no. 4, pp. 249-253, 1994.

[2] L. Yan and F. Zhijie, "The application of SSD deep learning algorithm in manipulator grasping system," Modular Machine Tool and Automated Processing Technology, vol. 556, no. 6, pp. 72-75, 2020.

[3] G. Jun, Z. Xin, Z. Zikang, Y. Xinhao, and L. Shaoze, "Application of deep learning in oil and gas seismic exploration," China Petroleum and Chemical Standards and Quality, vol. 39, pp. 88-92, 2019.

[4] W. Wang, C. Tieyong, Y. Zheng, and L. Feibin, "Lecture 5: application of deep learning in saliency detection," Military Communication Technology, vol. 37, no. 2, pp. 92-97, 2016.

[5] Y. Tao and Z. Xi, "Application of deep learning technology in the teaching of mechanical engineering testing technology," Education Modernization, vol. 7, no. 19, pp. 181-184, 2020.

[6] G. Jiachen, G. Lei, and L. Lulu, "Application of object detection algorithm based on deep learning in cold rolling surface defect detection," Metallurgical Automation, vol. 43, pp. 25-28+33, 2019.

[7] Y. Shen, J. Yang, and Q. Shengchang, "Application of deep learning algorithm in intelligent equipment," Equipment Machinery, vol. 4, no. 7-9, p. 18, 2017.

[8] S. Huansheng, Z. Xiangqing, Z. Baofeng, and Y. Teng, "Vehicle target detection in complex scenes based on deep learning methods," Application Research of Computers, vol. 35, no. 4, pp. 316-319, 2018

[9] W. Lu, "Application of deep learning in the field of high energy physics," Physics, vol. 46, no. 9, pp. 597-605, 2017.

[10] L. Xiaodong and N. Haoran, "Application of deep learning technology in disciplinary fusion research," Frontiers of Data and Computing Development, vol. 2, pp. 103-113, 2020.

[11] W. Shunzhi, "Research on the application of deep learning method based on GWS platform_- taking a block of Daqing Oilfield as an example," Information System Engineering, vol. 313, no. 1, pp. 83-84+87, 2020.

[12] W. Hao, Y. Jiayong, F. Guangming, and W. Yu, "Application status and prospects of deep learning in geophysics," Progress in Geophysics, vol. 35, pp. 256-269, 2020.

[13] Y. Hong, L. Guoye, L. Yan, X. Rui, W. Qian, and W. Chengbin, "Research and application of deep learning in laboratory 
medicine," Chinese Journal of Laboratory Medicine, vol. 42, no. 12, pp. 1063-1066, 2019.

[14] T. Chunxia, L. Wenhua, and L. Jing, "Analysis of rare earth elements," Analysis Laboratory, vol. 37, no. 2, pp. 222-248, 2018.

[15] T. Qinghua, "Plant physiological effects of rare earth elements," Journal of Jilin Teachers College of Engineering and Technology, vol. 32, no. 2, pp. 87-88, 2016.

[16] Z. Huaiwei, Z. Xinyao, L. Yang, T. Xiao, and L. Xingguo, "Progress in the application of rare earth elements in hydrogen storage materials," Journal of the Chinese Rare Earth Society, vol. 34, no. 1, pp. 1-10, 2016.

[17] T. Chunxia, "Application progress in the analysis of rare earth elements by mass spectrometry," Analytical Laboratory, vol. 35, no. 12, pp. 1477-1482, 2016.

[18] H. Zhi, H. Qun, Y. Hong, J. Xiaoping, and R. Yuansheng, "Effect of trace Sm addition on microstructure and mechanical properties of AZ61 magnesium alloys," Rare Metal Materials and Engineering, vol. 45, no. 9, pp. 2275-2281, 2016.

[19] X. Mingjie, M. Shengming, and L. Chengsheng, "The geochemical characteristics of trace and rare earth elements of the Zhaojikou lead-zinc deposit in Anhui and discussion on the genesis of the deposit," Geology and Mineral Surveying, vol. 3, no. 2, pp. 160-164, 2020.

[20] L. Kun, T. Chaoyang, L. Jinsong, C. Yingxiong, and L. Fei, "Sources of ore-forming materials from the Xionao lead-zinc deposit in Songtao, Qiandong: rare earth elements and carbon, oxygen, sulfur, and lead isotopic constraints," Journal of Guilin University of Technology, vol. 38, no. 3, pp. 365-376, 2018.

[21] L. Wu, L. Yibo, J. Wang, W. Liangying, Z. Nan, and W. Na, "Study on the pretreatment method for the determination of rare earth elements in manganese ore by high-pressure closed digestion-inductively coupled plasma mass spectrometry," Rock and Mineral Testing, vol. 37, no. 6, pp. 637-643, 2018.

[22] W. Zhonghua, S. Peishi, Z. Ping et al., "The strengthening effect of rare earth elements on the simultaneous desulfurization and denitrification of biological tower flue gas," Environmental Engineering Journal, vol. 10, no. 4, pp. 321-327, 2016.

[23] L. Zheng and Z. Jiayi, "The effect of different rare earth elements on the primary phase morphology of semi-solid aluminum alloy in chaotic convection," Chinese Journal of Mechanical Engineering, vol. 52, no. 16, pp. 77-85, 2016.

[24] F. Wang, G. He, S. Xiaoming, Y. Yang, and Z. Taiping, "Study on the carrier of rare earth elements in rare earth-rich deep-sea sediments in the Pacific," Acta Petrologica Sinica, vol. 32, no. 7, pp. 2057-2068, 2016.

[25] W. Peipei, L. Xiao, and S. Weijiao, "Determination of rare earth elements in geological samples by microwave digestioninductively coupled plasma mass spectrometry," Chinese Journal of Analysis Laboratory, vol. 35, no. 2, pp. 235-240, 2016.

[26] G. Jiangfeng, Y. Duoxi, C. Jian, and C. Ping, "Geochemistry and geological genesis analysis of rare earth elements in the coal of Chongqing Longtan Formation," Earth Science Frontiers, vol. 23, no. 3, pp. 51-58, 2016.

[27] Y. Yang, L. Dexian, D. Wang et al., "Distribution characteristics and influencing factors of dissolved rare earth elements in surface water around ion-adsorbed rare earth mining areas," Earth Science Frontier, vol. 24, no. 5, pp. 172-181, 2017.

[28] L. Shiyou, C. Yingbin, Z. Guowei, W. Yanqing, and H. Ye, "Geochemical characteristics and significance of rare earth elements in the fourth member of Leikoupo Formation in West
Sichuan Depression, Sichuan Basin," Petroleum Geology \& Experiment, vol. 39, no. 1, pp. 94-98, 2017.

[29] J. Biao, G. Wu, C. Yuchuan et al., "Characteristics of trace rare earth elements in Shuangianzishan silver polymetallic deposit in Balinzuoqi, Inner Mongolia and its genetic constraints," Acta Geology, vol. 92, no. 4, pp. 769-786, 2018.

[30] C. Xinxin, H. Song, and L. Zheng, "Influence of rare earth elements on the biological effects of sh_2 sweet corn seed germination," Journal of the Chinese Rare Earth Society, vol. 34, no. 2, pp. 229-234, 2016. 